\title{
Peripheral Nerve Injury
}

National Cancer Institute

\section{Source}

National Cancer Institute. Peripheral Nerve Injury. NCI Thesaurus. Code C101250.

Sensory or motor loss of peripheral nerve function due to a traumatic event. (ACC) 